\title{
Elastic scattering dynamics of cavity polaritons: Evidence for time-energy uncertainty and polariton localization
}

\author{
Langbein, Wolfgang Werner; Hvam, Jørn Märcher
}

Published in:

Physical Review Letters

Link to article, DOI:

10.1103/PhysRevLett.88.047401

Publication date:

2002

Document Version

Publisher's PDF, also known as Version of record

Link back to DTU Orbit

Citation $(A P A)$ :

Langbein, W. W., \& Hvam, J. M. (2002). Elastic scattering dynamics of cavity polaritons: Evidence for timeenergy uncertainty and polariton localization. Physical Review Letters, 88(4), 047401.

https://doi.org/10.1103/PhysRevLett.88.047401

\section{General rights}

Copyright and moral rights for the publications made accessible in the public portal are retained by the authors and/or other copyright owners and it is a condition of accessing publications that users recognise and abide by the legal requirements associated with these rights.

- Users may download and print one copy of any publication from the public portal for the purpose of private study or research.

- You may not further distribute the material or use it for any profit-making activity or commercial gain

- You may freely distribute the URL identifying the publication in the public portal 


\title{
Elastic Scattering Dynamics of Cavity Polaritons: Evidence for Time-Energy Uncertainty and Polariton Localization
}

\author{
Wolfgang Langbein \\ Experimentelle Physik EIIb, Universität Dortmund, Otto-Hahn-Strasse 4, D-44227 Dortmund, Germany \\ Jørn M. Hvam \\ Research Center COM, The Technical University of Denmark, Building 349, DK-2800 Kogens Lyngby, Denmark
}

(Received 24 September 2001; published 15 January 2002)

\begin{abstract}
The directional dynamics of the resonant Rayleigh scattering from a semiconductor microcavity is investigated. When optically exciting the lower polariton branch, the strong dispersion results in a directional emission on a ring. The coherent emission ring shows a reduction of its angular width for increasing time after excitation, giving direct evidence for the time-energy uncertainty in the dynamics of the scattering by disorder. The ring width converges with time to a finite value, a direct measure of an intrinsic momentum broadening of the polariton states localized by multiple disorder scattering.
\end{abstract}

DOI: 10.1103/PhysRevLett.88.047401

In resonant secondary emission of light (SE) from solid state media, scattering by static disorder leads to coherent resonant Rayleigh scattering (RRS), while scattering with other quasiparticles (e.g., phonons) leads to an incoherent emission. For a semiconductor quantum well $(\mathrm{QW})$ the SE does not depend significantly on the emission direction due to the small exciton dispersion within the optically accessible in-plane wave vectors, which is typically below $100 \mu \mathrm{eV}$ and smaller than the exciton linewidth. Oppositely, polaritonic states have a strong dispersion due to their photonic content and show a distinct dependence of the SE on its direction, as is observed in multiple QW structures $[1,2]$. In semiconductor quantum well microcavities (MC), cavity polaritons are formed [3] with a very steep dispersion at small in-plane wave vectors. Therefore the energy conservation in the RRS translates into a directional RRS when the polariton linewidth is smaller than the polariton dispersion within the optically accessible wavevector region. Accordingly, an annular RRS has been predicted $[4,5]$ and an enhanced RRS on a ring was found experimentally [6,7]. The directional dynamics of the RRS thus allows to monitor the time evolution of the scattering between the polaritons without a time-bandwidth limit in the detection, since the polariton eigenenergy can be determined from the emission direction.

In this Letter, we investigate the directional RRS dynamics from a MC using two-dimensional ultrafast timeresolved imaging. We find that the RRS occurs on a ring of constant in-plane momentum $\hbar|\mathbf{k}|$, having a width that decreases with time, in agreement with the time-energy uncertainty in the elastic scattering, down to a minimum width due to polariton localization by multiple scattering. The investigated sample [8] consists of an MBE-grown $25 \mathrm{~nm} \mathrm{GaAs} / \mathrm{Al}_{0.3} \mathrm{Ga}_{0.7} \mathrm{As}$ single quantum well placed in the center of a $\lambda$ cavity with AlAs $/ \mathrm{Al}_{0.15} \mathrm{Ga}_{0.85} \mathrm{As}$ Bragg reflectors of 25 (16) periods at the bottom (top). The sample was held in a helium cryostat at a temperature of $T=5 \mathrm{~K}$. The resonant
PACS numbers: 78.66.Fd, 71.36.+c

SE was excited by Fourier-limited optical pulses from a mode-locked Ti:sapphire laser of 1 ps pulse length at $76 \mathrm{MHz}$ repetition rate. The pulses were focused on the sample to a diffraction-limited spot of $80 \mu \mathrm{m}$ diameter and a $|\mathbf{k}|$ width of $0.035 \mu \mathrm{m}^{-1}$. The excitation intensity was $\approx 1 \mathrm{~nJ} / \mathrm{cm}^{2}$, corresponding to a polariton density of below $10^{9} \mathrm{~cm}^{-2}$. This is close to the lowdensity limit for polariton-polariton scattering. Spectrally resolved data were taken using a spectrometer and a CCD camera with a resolution of $20 \mu \mathrm{eV}$. Time-resolved data were taken using a spectrometer and a synchroscan streak camera with a time resolution of $3 \mathrm{ps}$. All widths given in the paper are full widths at half maximum. The sample was characterized by reflection measurements reported in Refs. [8] and [9]. The use of a wide GaAs quantum well leads to a negligible inhomogeneous broadening of the QW exciton due to the small effect of interface roughness. The exciton-photon coupling in the MC creates three polariton resonances from the heavy-hole exciton, light-hole exciton, and cavity mode. The mixing is well described by a three coupled-oscillator model [8]. From the measured polariton linewidths a cavity linewidth of $0.26 \mathrm{meV}$ and an excitonic broadening of $0.13 \mathrm{meV}$ were inferred [9]. The measured and calculated polariton dispersions for $-8.8 \mathrm{meV}$ detuning between the heavy-hole exciton and the cavity mode are shown in Fig. 1. The strong dispersion of the lower polariton branch (LPB) within the experimental wave-vector range is visible, and well described by the calculated dispersion. In the following, we discuss the SE at this detuning. The directionally resolved, time-integrated SE intensity is shown in Fig. 2 for an incident in-plane wave vector $\mathbf{k}=1.85 \mu \mathrm{m}^{-1} \hat{e}_{[110]}$ along the [110] direction. The strong negative detuning was chosen to avoid resonant contributions of bound exciton states present 1 to $4 \mathrm{meV}$ below the heavy-hole exciton. The qualitative dynamics of the initial RRS reported in the following was found also at more positive detunings. The shadow visible in 


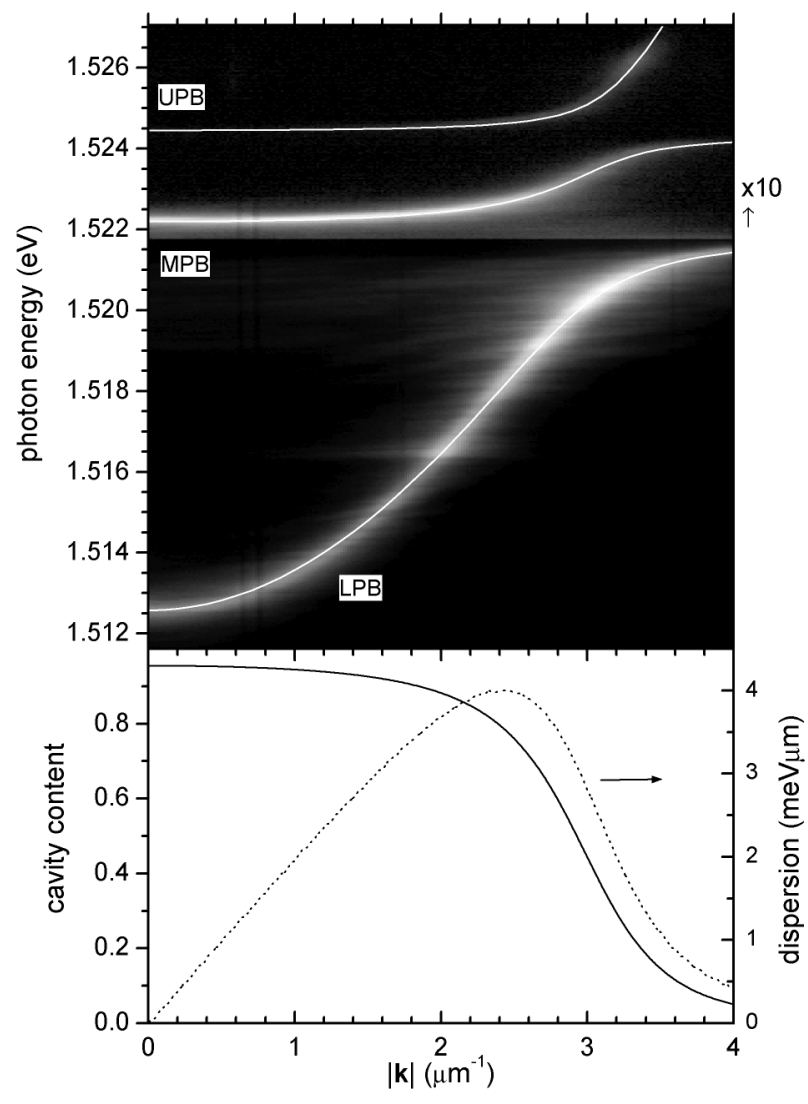

FIG. 1. Measured and calculated dispersion of the polariton modes at $-8.8 \mathrm{meV}$ detuning. Top: Cross-linear polarized SE for excitation in the UPB at $|\mathbf{k}|=3.3 \mu \mathrm{m}^{-1}$ (logarithmic gray scale over 3 orders of magnitude). Solid lines: Results from a three oscillator model [8] using $E_{\text {cav }}=1.51303 \mathrm{eV}$, $E_{\mathrm{hh}}=1.52185 \mathrm{eV}, \quad E_{\mathrm{lh}}=1.52433 \mathrm{eV}, \quad \Omega_{\mathrm{hh}}=1.8 \mathrm{meV}$, $\Omega_{\mathrm{lh}}=1.1 \mathrm{meV}, M_{\mathrm{hh}}=M_{\mathrm{lh}}=0.4 m_{\mathrm{e}}$, and $n_{\mathrm{cav}}=3.5$. Bottom: Calculated cavity content (solid) and dispersion slope (dashed) of the LPB.

the right side of Fig. $2 \mathrm{a}$ is due to a beam block for the specular reflection, which is 4 to 5 orders of magnitude more intense than the RRS. Strong stripelike scattering occurs along the [110] and [110] directions. We attribute this to scattering of the photonic part of the polariton by disorder in the Bragg mirrors. The well-defined scattering directions imply a correlation length of the scattering disorder comparable to the spot size. Its origin is the crosshatched disorder forming in lattice-mismatched growth [10] due to interfacial misfit dislocations. It is present in the Bragg mirrors of $0.2 \%$ lattice mismatch and several micrometer thickness, as we confirmed by near-field images (not shown).

Apart from the stripelike scattering, a narrow annular emission of nearly rotational symmetry is observed. It shows strong fluctuations on a small directional scale, i.e., speckles, which demonstrate its dominant coherent nature. The emission is copolarized to the excitation, showing that the disorder scattering is largely polarization conserving, as expected both for scattering by dielectric roughness of the Bragg mirrors, and for RRS in QWs with small inhomogeneous broadening [11]. The spectrally resolved
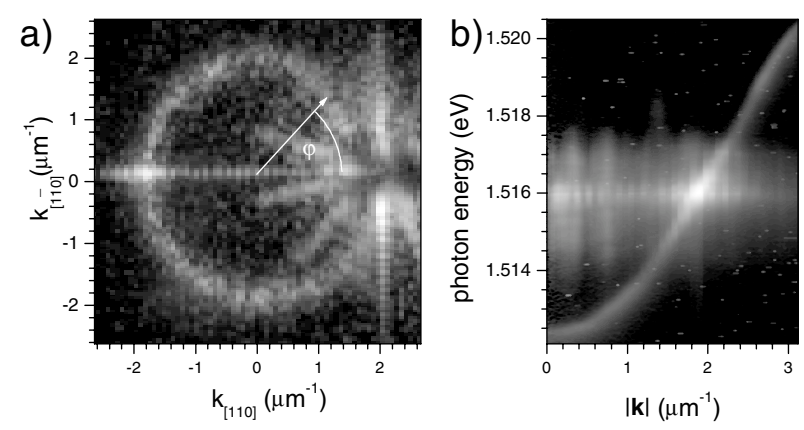

FIG. 2. SE imaging for excitation of the LPB at $\mathbf{k}_{0}=1.85 \mu \mathrm{m}^{-1} \hat{e}_{[110]}$ on a logarithmic gray scale over 3 orders of magnitude. (a) Spectrally integrated; (b) spectrally resolved and averaged over the azimuthal angle $\varphi$.

imaging (Fig. 2b) shows a nearly linear dispersion of the excited RRS, and some weak nonresonant emission on the LPB. In the following we concentrate on the RRS ring. To measure the two-dimensional dynamics of the polariton scattering, we developed an ultrafast imaging technique which allows us to measure the directionally resolved dynamics of the SE in two-dimensional $\mathbf{k}$ space with 3 ps temporal resolution by combining multiple streak-camera measurements. In Fig. 3 we show two-dimensional $\mathbf{k}$ space images of the RRS intensity for different times after excitation, as indicated. It is clearly seen that at early times the RRS ring is rather broad in $|\mathbf{k}|$, and narrows considerably up to $10 \mathrm{ps}$.

The RRS is due to polarization transfer from the initially excited polariton state $P_{\mathbf{k}_{0}}$ to polariton states $P_{\mathbf{k}}$ at different quasimomenta $\mathbf{k}$ by disorder scattering. We can in good approximation assume that the radiative lifetime of all participating states is equal, so that the radiative decay does not influence the scattering between the states. To visualize only the internal polariton scattering dynamics, we thus correct the RRS intensity for the polariton radiative decay. The resulting RRS intensity as function of $|\mathbf{k}|$ is shown in the upper inset of Fig. 4 for different times. The curves are azimuthal averages over a few hundred speckles of the two-dimensional data. A strong narrowing of the ring with increasing time can be seen, which we interpret in the following.

The disorder leading to the RRS does not depend significantly on the scattering wave vector [5], as is evident from the nearly circular symmetry of the RRS intensity. The differences of the $P_{\mathbf{k}}$ polarization amplitudes are thus determined by the different mismatch of the polariton eigenenergies, i.e., $E_{\mathrm{LPB}}(|\mathbf{k}|)-E_{\mathrm{LPB}}\left(\left|\mathbf{k}_{0}\right|\right)$, which is given by $|\mathbf{k}|$. At early times after excitation of the $P_{\mathbf{k}_{0}}$ polarization, its energy is not well defined due to timeenergy uncertainty. It can thus be scattered to $P_{\mathbf{k}}$ of a large $|\mathbf{k}|$ range. With increasing time, the energy uncertainty of $P_{\mathbf{k}_{0}}$ is decreasing and accordingly also the $|\mathbf{k}|$ range of the final states $P_{\mathbf{k}}$ in the scattering. The smaller the energy mismatch of $P_{\mathbf{k}}$, the longer is the scattering time, allowing for larger amplitudes to be transferred. This is an extreme case of non-Markovian behavior due to the principally 


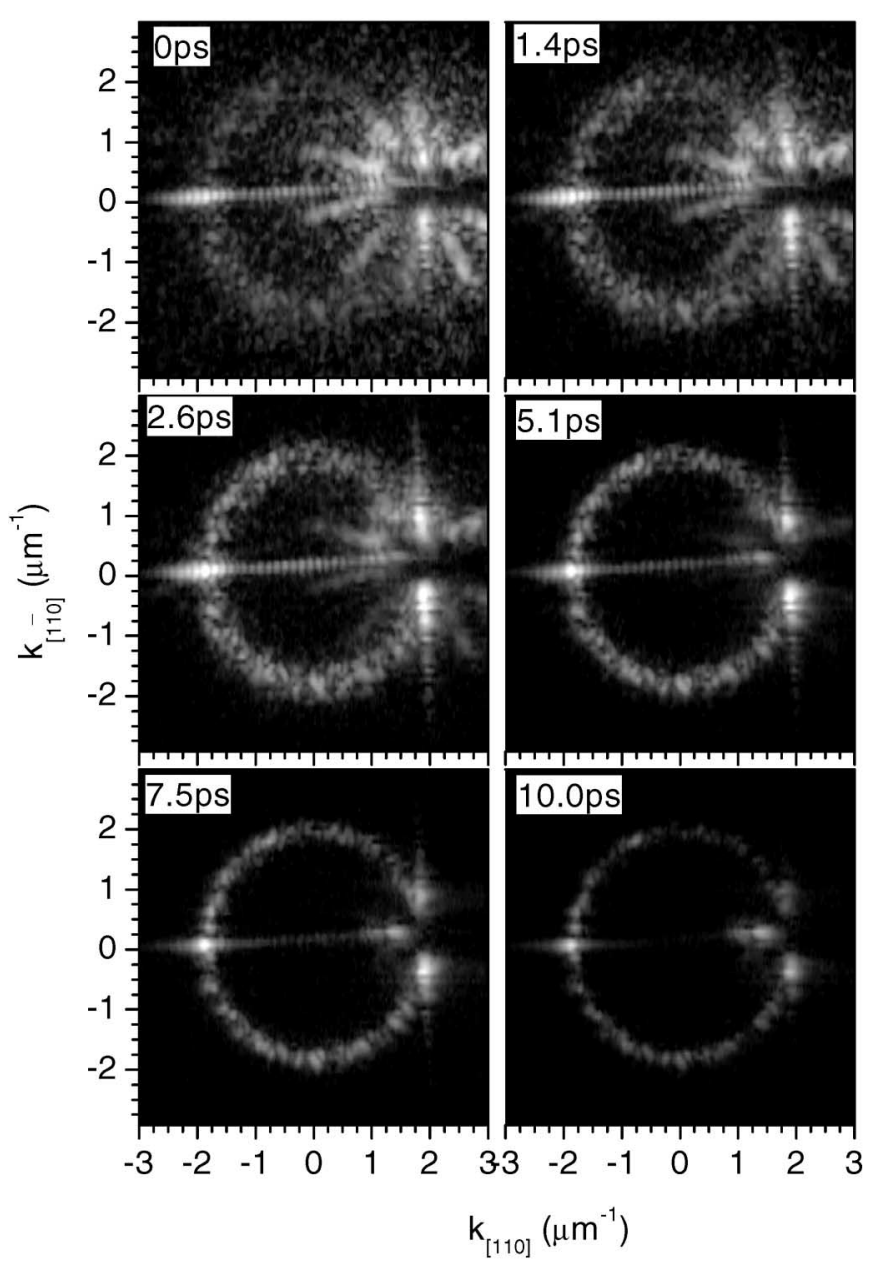

FIG. 3. Directional images of the SE intensity for times as indicated after resonant excitation of the LPB at $\mathbf{k}_{0}=1.85 \mu \mathrm{m}^{-1} \hat{e}_{[110]}$, shown on a fixed logarithmic gray scale spanning 3 orders of magnitude. The star shape around zero time is an artifact due to the excitation.

infinite memory time of scattering by static disorder [12]. The influence of the energy uncertainty of the $P_{\mathbf{k}_{0}}$ polarization on the scattering dynamics can be modeled using the time-bandwidth product $\Delta E \Delta t \approx 0.88 \mathrm{~h}$ of a square time-window $\Delta t$ where $\Delta E$ is the width of the energy distribution. The measured ring width dynamics is reproduced by this model (see solid line in middle Fig. 4) only at early times, while later the experimental width is underestimated. The experimental resolution, given by the $\mathbf{k}$ width of the excitation $\left(0.035 \mu \mathrm{m}^{-1}\right)$, cannot account for this deviation. Since the energy-time uncertainty is valid at all times, one has to conclude that the polariton eigenstates are not pure $\mathbf{k}$ eigenstates, but are broadened in $\mathbf{k}$ space. For long times, the width of the ring is then not converging to zero, but to the finite $\mathbf{k}$ width of excited polariton eigenstates. The $\mathbf{k}$ broadening of the polariton eigenstates has to be considered both in their excitation by the external light pulse, and in the reemission of the RRS. The observed RRS dynamics can thus also be envisioned as being due to interference in the emission from the ensemble of excited polariton

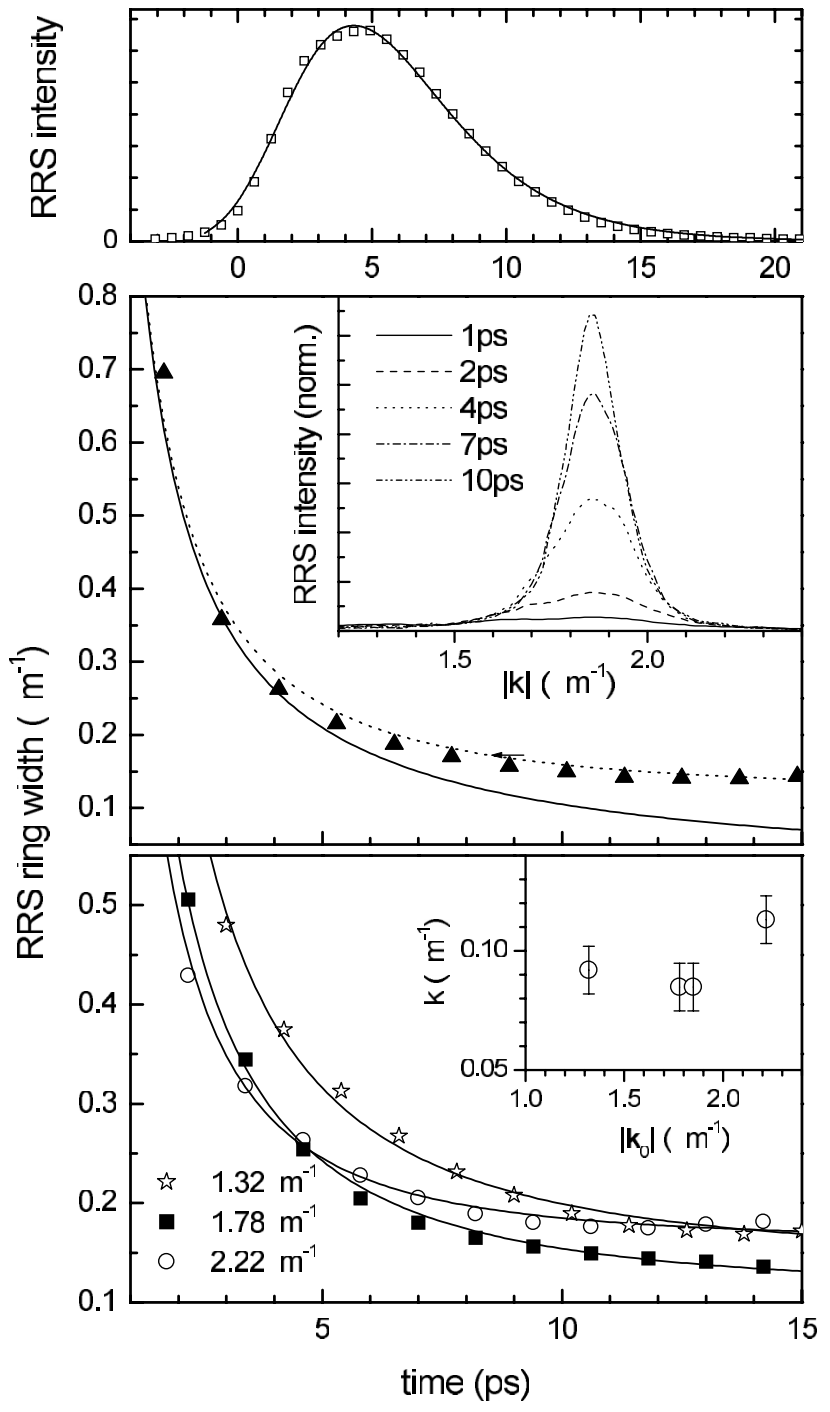

FIG. 4. Top: Time-resolved RRS intensity. The line is a fit using Eq. (2). Middle and bottom: Width of the RRS ring (symbols) as a function of time after resonant excitation of the LPB. Middle: $\left|\mathbf{k}_{0}\right|=1.85 \mu \mathrm{m}^{-1}$. The solid (dashed) line is a fit using Eq. (1) with $\Delta k_{\mathrm{LPB}}=0(0.09) \mu \mathrm{m}^{-1}$. Inset: Lifetimecorrected RRS radial distributions for different times, as indicated. Bottom: Different $\left|\mathbf{k}_{0}\right|$ as indicated, with fits. Inset: Fitted $\Delta k_{\mathrm{LPB}}$ as function of $\left|\mathbf{k}_{0}\right|$.

eigenstates, which change their relative phases with time due to their distribution of eigenenergies. In the modeling we include the $\mathbf{k}$ broadening of the polariton eigenstates adding to the width due to time-energy uncertainty an intrinsic polariton $|\mathbf{k}|$ width $\Delta k_{\mathrm{LPB}}$ in excitation and in emission in a Gaussian sum. The time-resolved width of the RRS is then described by

$$
\Delta k_{\mathrm{RRS}}(\Delta t)=\sqrt{\left(\frac{0.88 h}{\Delta t \partial_{k} E_{\mathrm{LPB}}}\right)^{2}+2 \Delta k_{\mathrm{LPB}}^{2}} .
$$

The experimental data are in good agreement with this model using the measured dispersion slope $\partial_{k} E_{\mathrm{LPB}}$ (see Fig. 1) and $\Delta k_{\mathrm{LPB}}=0.092 \pm 0.02 \mu \mathrm{m}^{-1}$. The value of $\Delta k_{\mathrm{LPB}}$ gives a lower limit for the spatial extend of the 
polariton eigenstates of $\Delta x$ of about $30 \mu \mathrm{m}$. This large localization length compared to bare QW excitons is evidence for the strong motional narrowing of the polaritons.

The ring width dynamics are well described by Eq. (1) for all investigated $\left|\mathbf{k}_{0}\right|$ using the respective dispersion slopes $\partial_{k} E_{\mathrm{LPB}}\left(\left|\mathbf{k}_{0}\right|\right)$. The fitted intrinsic polariton widths $\Delta k_{\mathrm{LPB}}$ are shown in the lower inset of Fig. 4.

The directionally averaged RRS ring intensity (see Fig. 4, top) has a delayed maximum and a subsequent exponential decay. The dynamics can be modeled taking the inhomogeneously broadened distribution of excited polariton states, similar to the QW case [13], for which the RRS intensity $I(t)$ is [14]

$$
I(t) \propto \exp \left(-2 \gamma_{r} t\right)\left[1-\exp \left(-\sigma^{2} t^{2}\right)\right]
$$

with the radiative decay rate $2 \gamma_{r}$ and the inhomogeneous broadening $\hbar \sigma \sqrt{8 \ln 2}$. The dynamics is fitted by an inhomogeneous broadening of $0.28 \mathrm{meV}$, using the nominal radiative lifetime of 3 ps calculated from the photonic content and the cavity lifetime. The deduced inhomogeneous broadening is similar to the previously deduced polariton dispersion over the polariton $|\mathbf{k}|$ width $\Delta k_{\mathrm{LPB}} \partial_{k} E_{\mathrm{LPB}}$, a confirmation of the RRS description by localized polariton eigenstates.

Multiple scattering of polaritons, which leads to polariton localization, should also lead to an enhanced backscattering, as it was observed already in a time-integrated experiment [7] on a MC sample with a polariton radiative decay slower than the disorder scattering. Our sample is in the opposite regime, leading to the dominance of single scattering in time-integrated RRS, which does not show enhanced backscattering. However, in time-resolved RRS, we can differentiate between single and multiple scattering of polaritons, which occurs at early and late times, respectively. The dependence of the RRS intensity on the azimuthal angle is shown in Fig. 5 for excitation at two different $\left|\mathbf{k}_{0}\right|$. The observed strong fluctuations are due to insufficient speckle averaging over the RRS ring, which contains only a few speckles in the radial direction. At early times $(0-5 \mathrm{ps})$, we observe forward scattering, while at late times an enhanced backscattering evolves, which has a large angular width similar to previous observations [7]. The corresponding large azimuthal $\mathbf{k}$ width of the eigenstates as compared to the radial one can be explained by the strong radial dispersion of the polariton, which is absent in the azimuthal direction. We also notice that the back-scattering peak is slightly shifted from $\pi$ to larger angles, which might be related to the small cavity wedge present in the sample, which leads to a polariton energy decreasing along the direction $\frac{3}{2} \pi$.

In conclusion, we have shown that the RRS from microcavity polaritons occurs on a ring in momentum space with a directional broadening decreasing with increasing time after excitation. This is a direct evidence for the energy-time uncertainty in the disorder scattering. The broadening of the RRS ring converges at long times to a finite value given by the momentum broadening of

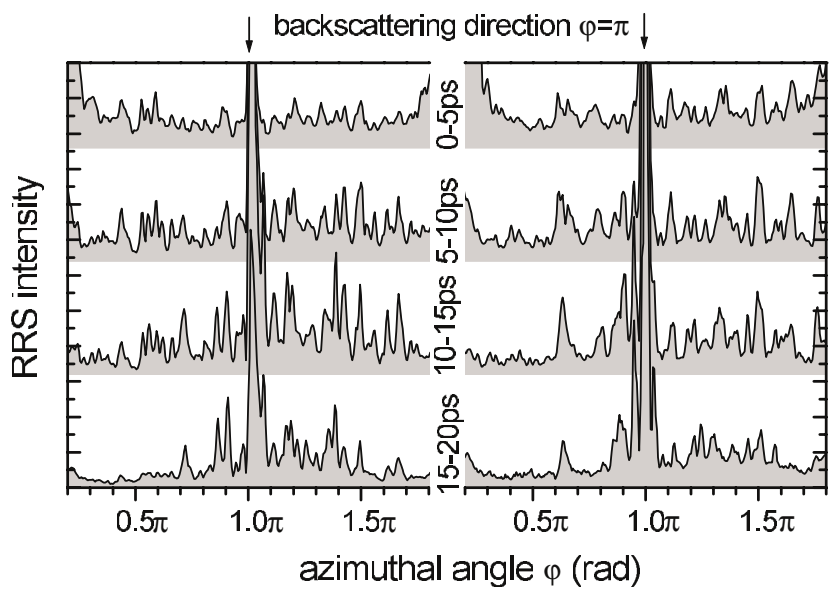

FIG. 5. Normalized RRS ring intensity as a function of the azimuthal angle $\varphi$ at different times, as indicated. The left (right) data are taken for excitation of the LPB at $\left|\mathbf{k}_{0}\right|=$ $2.22(1.85) \mu \mathrm{m}^{-1}$, respectively.

the polariton eigenstates, a sign of polariton localization. The dynamics of the RRS intensity and the observed enhanced backscattering are in agreement with such a polariton localization by multiple disorder scattering. Recent theoretical treatments [4,5] described MC RRS in a single scattering approximation, in which the present observations cannot be explained. A theoretical treatment of multiple scattering in MC, taking into account both QW and dielectric disorder, is thus highly desirable.

The authors want to acknowledge J. R. Jensen for the growth of the MC and R. Zimmermann and P. Borri for discussions. The sample was grown at III-V Nanolab, a joint laboratory between Research Center COM and the Niels Bohr Institute, Copenhagen University. This work was in part supported by the German Science Foundation (DFG) within the "Schwerpunktprogramm Quantenkohärenz in Halbleitern."

[1] G. Malpuech, A. Kavokin, W. Langbein, and J. M. Hvam, Phys. Rev. Lett. 85, 650 (2000).

[2] M. Hübner et al., Phys. Rev. Lett. 83, 2841 (1999).

[3] C. Weisbuch, M. Nishioka, A. Ishikawa, and Y. Arakawa, Phys. Rev. Lett. 69, 3314 (1992).

[4] D. M. Whittaker, Phys. Rev. B 61, R2433 (2000).

[5] A. V. Shchegrov, J. Bloch, D. Birkedal, and J. Shah, Phys. Rev. Lett. 84, 3478 (2000).

[6] T. Freixanet et al., Phys. Rev. B 60, R8509 (1999).

[7] R. Houdré et al., Phys. Rev. B 61, R13 333 (2000).

[8] J. R. Jensen, P. Borri, W. Langbein, and J. M. Hvam, Appl. Phys. Lett. 76, 3262 (2000).

[9] P. Borri, J. R. Jensen, W. Langbein, and J. M. Hvam, Phys. Rev. B 61, R13 377 (2000).

[10] K. H. Chang et al., J. Appl. Phys. 67, 4093 (1990).

[11] H. Stolz, D. Schwarze, W. von der Osten, and G. Weimann, Phys. Rev. B 47, 9669 (1993).

[12] R. Zimmermann, Nuovo Cimento D 17D, 1801 (1995).

[13] S. Haacke et al., Phys. Rev. Lett. 78, 2228 (1997).

[14] W. Langbein, J. M. Hvam, and R. Zimmermann, Phys. Rev. Lett. 82, 1040 (1999). 